\section{Combining Interaction Design and Gaming Technologies for the Development of Interactive Archaeological Content Presentation Systems}

Ioannis Deliyannis ${ }^{1^{*}}$ and Georgios Papaioannou ${ }^{2}$

${ }^{1}$ Department of Audio and Visual Arts, Ionian University, Greece

${ }^{2}$ Department of Audiovisual Arts, Ionian University, Corfu, Greece

*Corresponding author: Ioannis Deliyannis, Department of Audio and Visual Arts, lonian University, Plateia Tsirigoti 7, Corfu, Greece, Tel: +306936747175; E-mail: yiannis@ionio.gr

Received date: November 24, 2016; Accepted date: December 16, 2016; Publication date: January 01, 2017

Copyright: (c) 2017 Deliyannis I, et al. This is an open-access article distributed under the terms of the Creative Commons Attribution License; which permits unrestricted use; distribution; and reproduction in any medium; provided the original author and source are credited.

\begin{abstract}
Our main objective is to produce state-of-the-art edutainment and serious game end-systems, which satisfy the requirements of all three parties involved in the development process: content experts, end-users and application developers. Their requirements are often cross disciplinary, as each party involved in the process requires solutions to a number of problems which need to be answered in a systematic and complete manner. The ultimate goal of this process is to introduce an efficient, extendable and aesthetically pleasing end-system. In order to achieve these goals, we address and attempt to resolve the most common presentation design issues that arise during the process of interaction design. Completion of this process enables the actual system development to commence with a precise and complete specification of content features and system characteristics.
\end{abstract}

Keywords: Interaction design; Multimedia; Gaming technology; Edutainment; Archaeological content

\section{Introduction}

Our previous work [1-5] has demonstrated that it is possible to develop applications and games featuring multiple interactive scenaria, which employ the same set of information markers and triggers in order to cover unique presentation requirements [6]. It was shown how this approach essentially extends the interactivity of a system and provides the capability to offer multiple or scalable interaction scenaria using the same information space [7]. The applicability of the proposed method has already been tested in practice via the development of interactive artwork, games and edutainment applications [8], using state of the art mobile platforms and augmented reality environments [9]. These systems combine real-life and virtual content, cover complex content presentation demands and are designed to adapt according to varying user needs and variable hardware specifications. Note that it is common for such systems to be used synchronously by different user groups, including young [10] and older visitors [11], enabling them to interact with the content using customised narration that suits their personal profile. In addition, users access and interact with the content using personal hardware and network access providers [12].

A requirement for the design of such systems is to first define the information space [8] and decide on system functionality in contrast to intended audience(s). Interaction design is clearly the most important process that affects both end-system quality and user experience. Interestingly, the Association for Computing Machinery (ACM) in 2012 classified interaction design as an autonomous research field, beyond the boundaries of human-computer interaction (HCI). This development identifies clearly that interaction design is not only part of user experience and other human-computer interaction (HCI) fields. Consequently, our research discusses important interaction design and research issues such as game complexity, interactivity, multimedia content use, storytelling and overall game aesthetics.

In addition, multiple application modes are addressed covering the range between single-player to multiplayer and score-based games. Within the software context, our analysis presents the main game development technologies and platforms available today, focusing particularly on open source software, which may be employed under the intended purpose. Pieces of open source software include HTML-5, Augmented Reality Platforms, Multimedia and Game Authoring Environments, Virtual Reality and Social-Media-based platforms. Ultimately, the use of state-of-the-art hardware is addressed, where we discuss the adaptability of interactive content in order to cover multiple platforms, particularly when user-based hardware is employed in order to cover the presentation requirements and provide a unified game experience.

\section{Archaeological Content, Data Types and Interaction Requirements}

In this work we focus on the design of edutainment and serious games applications $[13,14]$. In this particular subject-area there exist multiple developmental paradigms [15-17], which are often adapted in order to cover specific content, system and user requirements [18]. Existing methodologies either utilise a generalised interaction design approach for all purposes, or focus system development on the technological platform capabilities [19-32]. Some choose to map learning and game mechanics in order to analyse the performance of the produced application [33], or create models of the end-system in order to predict its end-user performance [34]. In fact some of those approaches suffer from typical software system side effects. Some of those include limited end-system functionality, limited adaptability when new content is introduced, and the lack of flexibility when major changes need to be implemented within the end-system due to content extension and the inclusion of new features. This work presents a novel 
Page 2 of 5

methodology, which can simplify the game-design process, specify the desired content aspects and identify precisely the information space that will be communicated to the user. In other words, the process aids content experts (archaeologists in this case) to identify and easily select the desired gamification characteristics of the content, enabling developers to focus their interaction design methodologies on specific content presentation requirements. The implication of this process is that a developer is not limited by technology before the design of the system, enabling the combination of mixed media solutions for the gamification of content, a trend which is quite popular nowadays [35-40]. Clearly, this approach provides content experts with the freedom to design an interactive experience which is not limited by technological specifications. It also allows developers to choose the most appropriate software/hardware combination. Last but not least, it provides users with an adaptive state-of-the-art interactive experience, instead of a generalised multimedia product.

\section{Common content characteristics and data types}

The majority of archaeological multimedia systems designed to provide an information / learning experience to the user employ a wide range of content data types, similar to those employed by multimedia-based applications such as interactive TV [41-46]. In Table 1 we list the most common data types used in such systems.

\begin{tabular}{|c|c|c|c|}
\hline Data type & Use & Format & Interaction Requirements Complexity \\
\hline Text & Content & Plain / Encoded Formats & Low \\
\hline Text & Labelling & Plain / Encoded Formats & Low \\
\hline Image & Content & Compressed Image & Medium \\
\hline Image & Labelling & Compressed Image & Medium \\
\hline Audio & Content/Instructions/Interaction & Mono/Stereo/Binaural & Low/Medium \\
\hline 3D Model & Content/Effects/Narration & 3D formats / motion path & Medium \\
\hline Video & Content/Effects/Narration & Compressed Video & Medium \\
\hline Projection Mapping & Content/Narration & Compressed Video & Medium \\
\hline Augmented Reality & Content/Narration & AR Environment & Medium \\
\hline Virtual Reality World & Content/Environment Restoration & VR World, CAVE & High \\
\hline
\end{tabular}

Table 1: Media types.

The contents' intended use by the developer is contrasted against the typical formats and most importantly their system handling and processing requirements, which we refer to as "interaction requirements". Low-complexity media such as text, images and audio are considered as the basic building blocks for the more complex data types. However we refer to the content at a higher level, that of a "multimedia frame" [47], each combining one or more of the above content types. Multimedia frames are considered as information entities that can be engineered to refer to real-life entities. For example, in the case of archaeological content, we consider the case of a particular archaeological museum collection in Igoumenitsa, Northwestern Greece, consisting of excavated items originating from a kitchen in ancient Greece [48].

Each artefact can be represented using a multimedia frame, combining single media and multimedia information that may include: title, item description, history, location found, 3D model, video of the excavation site, images of the original state found, video of the restoration process, how it was used in the past, and all other information available for each particular item. In addition, a multimedia frame can combine direct information linking to lowerorder information or higher-order structures - like the collection itself. Clearly, Table 1 lists only the typical data types and not the hybrid combinations that a developer may employ in order to cover special presentation demands. This appears more often with multimedia content which is found in multiple formats: video-based documentaries, animated and interactive 3D models, mixed and virtual reality applications, augmented reality guides and presentations, projection mapping and immersive experiences utilising many of the human senses in the information/learning process.

\section{Interaction requirements and instructional design}

It is informative to examine the topic of interaction requirements, as the proposed system development approach focuses on the presentation of archaeological content. The development process consists of three distinct roles: content expert, user and developer. Our past research analysed their roles in the process of creating an augmented-reality tour that supports dynamic-dynamic interactive routes [49].

Interaction requirements must cover the demands of instructional design for the content domain. It is evident that instructional design is a key aspect of system development. System requirements analysis is therefore adjusted to cover the learning process. This comprises an integral part of the development process. In the literature it is evident that this also applies for multi-modal systems [50]. The proposed model maps the content in a graph-type structure after the requirements analysis is completed. It is interesting to examine how other instructional design models deal with their design and development stages. Take for example ADDIE, an Instructional Design (ID) model employed for the development of multimedia educational games using mobile phones [51]. In this case, developers employ userdriven evaluation cycles of the end-system. Other instructional design models including the Dick and Carey, Kemp's Instructional Design, Gagné's Nine Events of Instruction, Bloom's Learning Taxonomy, 
Kirkpatrick's 4 Levels of Training Evaluation and Cathy Moore's Action Mapping, also connecting the result to the learning process. The main difference between the above and our proposed model is that they include the user responses in their functional learning process, while in our case, the user responses are only embedded into the design process of each system version. Due to the advanced interactive features offered by the produced end-system, the user is able to switch presentation modes, essentially adjusting the learning process to suit their own timing and level, a process that was often found to remove the need for system re-engineering and updates.

The learning effects of such interactive learning modes have been measured and the results are very promising [52]. A content expert can define the learning objectives. In the case of archaeological content learning objectives may vary, depending on each user group. We have shown in previous work how this can be implemented via the selection of information subsets from the complete domain [7] for users with different demands. The system reveals the most appropriate set of information and the user is allowed to interact with it, enabling dynamic adjustment of content access.

From the user perspective, when a specific route is selected, then the starting node of the route is loaded, complete with links to the route's related node(s), and so on. This organisation is commonly described as a graph where nodes represent the information points. Arrows between nodes offer relevant user options that can be displayed at each node. In our work we have already described how multimedia frames can be interconnected [53]. Our past multimedia systems utilise a wide arrange of case studies presenting this technology in action, featuring dynamic interaction within complex multimedia content [54].

The developer focuses in a number of points: system functionality, ensuring that each navigational path is complete and informative; information accuracy, where each user is presented with the correct information at each stage; interaction adaptability, enabling the system to switch presentation modes when that is required, without repeating information which was presented before; and finally, usability and presentation aesthetics, a process enabling users with different backgrounds to employ the end-system and maximise its performance.

\section{System Development}

The process of system development demands the interaction requirements process to be completed and the main navigational routes through the content, including narration, to be decided. The outcome of the customised requirements-analysis approach described above (section 2.2) is used as a developmental basis. This information set encapsulates the essence of the end-system: the content organised in "multimedia frames", the interaction rules, its intended functionality in the form of pre-determined or open-ended scenaria and narratives. This structured multimedia graph is actively employed in order to cover multiple user requirements, a process which in some instances has allowed the automatic deployment of such systems directly from specification [49]. Uniformity of representation in frames is particularly important for archaeological content, as the resulting specification possesses a number of highly desirable characteristics. First, it is evident that the information space is well defined, as research information complements the archaeological findings. Secondly, many researchers may be active in information spaces, which are often complementary, offering different perspectives on the same archaeological findings. Therefore developers may collectively use content from different sources in a constructive manner, in order to provide multiple interactive learning experiences or educational games which re-use content and location-based triggers, featuring innovative scenaria. Multimedia databases are commonly employed for content organisation, enabling direct access by the end-system.

\section{Platforms and implementation}

Typically, web-based tools and time-based multimedia authoring environments are employed for the development of the end-system application. Today HTML-5 and Shockwave are the usual formats in which the content is delivered. HTML-based solutions as opposed to proprietary software offer more flexibility, particularly due to their standardization and accessibility in a wide variety of platforms. However the specification does not limit the developers to specific environments.

The process may be summarised as follows: the content expert organises the content and creates digital entities in the form of "multimedia frames" which will later be used as the information access points by the user. As the same information points can be employed for multiple purposes, in order to reduce developmental delays, it is important to include all the information that is available at the time. Then, user interaction requirements come to play. The content expert creates a set of rules, which enable the system to identify if a specific node can be accessible from all presentation states. Imagine the starting point of a guided tour where the user is presented with three different paths. The content expert chooses at this point to enable access to a "multimedia frame" containing each route starting point, complemented by a short description and a video presenting a summary of what the user will experience.

Take for example the guided tour case study of the Old Fortress Corfu, Greece [7]. Here, the augmented reality platform "Aurasma" was utilised in order to implement interaction using mobile phones and tablets. Multimedia frames containing historical information were linked to easily recognizable markers that the device's camera detects and displays the following information: directions to the next information points, historical information regarding the specific location, panoramic views of the location, map, old photographs and video, full archaeological information for that specific point.

Verification is a process that today requires extensive testing and effort, particularly when non-linear narrative paths are supported. Depending on the complexity of the implementation, a developer may choose to verify system performance using multiple methods. Realuser testing in the field is the most common method, where practical problems can also be revealed such as limited lighting for image recognition used in augmented reality, or the change of shadows in buildings that alters the stored image. Other issues that commonly arise include the audio performance in noisy environments, the lack of network connection that requires content caching to be supported, the GPS reception errors that provide erroneous readings and can cause to system underperform and the lighting conditions which affect what the user sees on screen. Finally, issues of usability and aesthetics are important, as those applications apply to wide audiences with a wide range of abilities. In order to provide acceptable interaction environments, we choose to employ the standard conventions for accessibility used in different environments, regarding access to audiovisual information, subtitles, the size of graphical objects and their contrast. 


\section{Conclusion and Future Research Directions}

In this work we have presented a number of issues that relate to interaction design for archaeological content. Our experience has shown that it is possible to design multimedia-enabled interactive systems featuring dynamic information switching, dynamic navigation, adjustable presentation of content, via the use of multimedia frames used to encapsulate both content and interaction design. There are many future research directions that we wish to pursue. These include the need for automatic system authoring based on the specification, a development that reduces the need for developers and can provide rapid prototyping methods that can be directly utilised by content experts. The issues of automatic interface production and aesthetics are also highly important, as those are the parts of the content that the users experience. Ultimately, we aim to reach a point where archaeological information will be standardised, enabling content experts to select and compile presentations dynamically.

\section{References}

1. Deliyannis I (2015) Interactive multimedia installation art development using recycled input and sensing devices. Int J Art Tech 9: 2.

2. Deliyannis I (2012) From interactive to experimental multimedia. Interactive Multimedia. pp: 3-12.

3. Deliyannis I, Simpsiri C (2008) Interactive multimedia learning for children with communication difficulties using the Makaton Method. International Conference on Information Communication Technologies in Education. pp: 10-12.

4. Deliyannis I, Kanellopoulos N (2008) Interactive communication methodology for social software and multimedia applications. IADIS International Conference. pp: 541-545.

5. Deliyannis I, Kermanidis KL (2007) Interactive Internet-based investigation of experimental and simulated multimedia datasets. IADIS International Conference.

6. Deliyannis I, Honorato D (2016) Developing augmented reality applications using branded authoring environments. In: Deliyannis I, Kostagiolas P, Banou Ch (eds.) Experimental multimedia systems for interactivity and strategic innovation. IGI Global: USA. pp: 73-101.

7. Deliyannis I, Papaioannou G (2016) Augmented reality edutainment systems for open-space archaeological environments: The case of the old fortress, Corfu, Greece. In: Deliyannis I, Kostagiolas P, Banou Ch (eds.) Experimental multimedia systems for interactivity and strategic innovation. IGI Global: USA. pp: 307-323.

8. Augusto JC, Zhang T (2014) Potential for augmented reality in education: An overview. Workshop Proceedings of the 10th International Conference on Intelligent Environments. Amsterdam, Netherlands.

9. Art C, Grasset R, Gruber L, Langlotz T, Mulloni A, et al. (2015) The history of mobile augmented reality.

10. Jarvin L (2015) Edutainment, games, and the future of education in a digital world. New Dir Child Adolesc Dev 147: 33-40.

11. Veloso A, Costa L (2015) Social network games in an ageing society: Codesigning online games with adults aged 50 and over. Information Systems and Technologies (CISTI), 2015 10th Iberian Conference on Information Systems and Technologies. pp: 1476-1481.

12. Deliyannis I, Kostagiolas P, Banou Ch (2016) Experimental multimedia systems for interactivity and strategic innovation.

13. Deliyannis I, Giannakoulopoulos A, Oikonomidou R (2013) Engineering and design issues in urban-oriented edutainment systems.

14. Deliyannis I, Giannakoulopoulos A, Varlamis I (2011) Utilising an educational framework for the development of edutainment scenarios. 5th European Conference on Games Based Learning. pp: 145-151.
15. Martinez JL, Alvareza S, Finat J, Delgado SJ, Finat J (2015) Augmented reality to preserve hidden vestiges in historical cities. A Case Study. Int Arch Photogram Rem Sens Spatial Inform Sci 40: 61-67.

16. Grammenos D, Zabulis X, Michel D, Sarmis T, Georgalis G, et al. (2011) Design and development of four prototype interactive edutainment exhibits for museums. 6th International Conference, UAHCI 2011, Orlando, FL, USA.

17. Ardito C, Costabile MF, Lanzilotti R (2009) Enhancing user experience while gaming in archaeological parks with cellular phones. Proceedings of the 8th International Conference on Interaction Design and Children, ACM New York, USA.

18. Chowaniec R (2013) New technologies in the process of educating about the archaeological heritage. Stara strona magazynu EduAkcja 5: 32-42.

19. De Paolis LT, Aloisio G, Celentano MG, Oliva L, Vecchio P (2011) A simulation of life in a medieval town for edutainment and touristic promotion.

20. Torrente J, Mera PL, Moreno-Ger P, Fernandez-Manjon B (2009) The Relationship between game genres, learning techniques and learning styles in educational computer games. Transactions on Edutainment II. pp: 1-18.

21. Sauvé L (2009) Design tools for online educational games: concept and application. Transactions on Edutainment II. pp: 187-202.

22. Ronchi AM (2009) Games and edutainment applications. eCulture: Cultural Content in the Digital Age. pp: 375-378.

23. Khine MS, Suja'ee MSB (2008) Core attributes of interactive computer games and adaptive use for edutainment. Transactions on edutainment I. pp: 191-205.

24. Rice J (2007) Assessing higher order thinking in video games. Journal of Technology and Teacher Education 15: 87-100.

25. Green M, McNeese MN (2007) Using edutainment software to enhance online learning. International Journal on E-Learning 6: 5-16.

26. Encarnação J (2007) Edutainment and serious games - games move into professional applications. International Conference on Technologies for E-Learning and Digital Entertainment. Springer Berlin-Heidelberg.

27. Egenfeldt-Nielsen S (2007) Third generation educational use of computer games. J Educ Multimed Hypermedia 16: 263-281.

28. Jang SH, Kye B, Kang Y (2006) A unified framework for u-edutainment development of using e-learning and ubiquitous technologies. KES. pp: 1163-1170.

29. Göbel S, Malkewitz R, Becker F (2006) Story pacing in interactive storytelling. International Conference on Technologies for E-Learning and Digital Entertainment. pp: 419-428.

30. Cai YY, Chan CW, Lu BF, Indhumathi C, Jiang Y (2006) VR-enabled bio edutainment. Technologies for E-Learning and Digital Entertainment, Berlin.

31. Wylin B, Desmet P (2005) How to integrate games or game elements in educational multimedia: a typology of educational game use. World Conference on Educational Media and Technology 1: 1194-1199.

32. Egenfeldt-Nielsen S (2005) Beyond edutainment: exploring the educational potential of computer games.

33. Arnab S, Lim T, Carvalho MB, Freitas SD, Louchart S, et al. (2005) Mapping learning and game mechanics for serious games analysis. Brit J Educ Technol 46: 391-411.

34. Zarraonandía T, Díaz P, Aedo I (2016) Modeling games for adaptive and personalized learning. The Future of Ubiquitous Learning. pp: 217-239.

35. Weedon A, Miller D, Franco CP, Moorhead D, Pearce S (2014) Crossing media boundaries: Adaptations and new media forms of the book. The International Journal of Research into New Media Technologies 20: 108-124.

36. Leiva J, Guevara A, Rossi C, Aguayo A (2014) Augmented reality and recommendation systems for groups. A new perspective on tourist destinations systems. Estudios y Perspectivas en Turismo 23: 40-59.

37. Dijck JV (2013) The culture of connectivity: A critical history of social media. 
Citation: Deliyannis I, Papaioannou G (2017) Combining Interaction Design and Gaming Technologies for the Development of Interactive Archaeological Content Presentation Systems. Int J Adv Technol 8: 178. doi:10.4172/0976-4860.1000178

Page 5 of 5

38. Solée RV, Valverde S, Casals MR, Kauffman SA, Farmer D, et al. (2013) The evolutionary ecology of technological innovations. Complexity 18 15-27.

39. Ramirez M, Ramos E, Cruz O, Hernandez J, Perez-Cordoba E, et al. (2013) Design of interactive museographic exhibits using Augmented reality. IEEE. pp: 1-6.

40. Minović M, Milovanović M, Starcevic D (2013) Literature review in game-based learning. Communications in Computer and Information Science 278: 146-154.

41. Deliyannis I (2013) Adapting interactive TV to meet multimedia content presentation requirements. International Journal of Multimedia Technology 3: 83-89.

42. Deliyannis I, Karydis I, Karydi D (2011) iMediaTV: open and interactive access for live performances and installation art. 4th International Conference on Information Law (ICIL2011), Botti Maria.

43. Deliyannis I, Karydis I (2011) Producing and broadcasting non-linear art-based content through open source interactive internet-TV. 9th International Interactive Conference on Interactive television.

44. Deliyannis I, Antoniou A, Pandis P (2009) Design and development of an experimental low-cost internet-based interactive TV station. 4th Mediterranean Conference on Information Systems (MCIS), Athens, Greece.

45. Cesar P, Chorianopoulos K (2009) The evolution of TV systems, content, and users toward interactivity. Foundations and Trends in HumanComputer Interaction 2: 279-374.

46. Chorianopoulos K (2008) User interface design principles for interactive television applications. Int J Hum-Comput Int 24: 556-573.
47. Deliyannis I (2011) Interactive multimedia systems for science and rheology: Interactive interrogation of complex rheological data using media-rich adaptive multimedia technologies.

48. Kanta-Kitsou A, Palli O, Anagnostou I (2008) Igoumenitsa archaeological museum. Greek Ministry of Culture: Igoumenitsa.

49. Deliyannis I, Papaioannou G (2014) Augmented reality for archaeological environments on mobile devices: a novel open framework. Mediterranean Archaeology and Archaeometry 14: 1-10.

50. Nogueira PA, Teófilo LF, Silva PB (2015) Multi-modal natural interaction in game design: a comparative analysis of player experience in a large scale role-playing game. J Multimodal User In 9: 105-119.

51. Norshahila I, Wan FWA (2015) Multimedia mobile learning application for children's education: The development of MFolktales. Asian Social Science 11: 203-215.

52. Umar IN, Aziz ZA (2015) The effects of multimedia with different modes of presentation on recitation skills among students with different selfregulated learning level. Social and Behavioral Sciences 197: 1962-1968.

53. Deliyannis I, Floros A, Webster MF (2009) An adaptive complex data investigation methodology using interactive multi-menus. Metadata and Semantics. pp: 393-402.

54. Deliyannis I, Harvey J, Webster MF (2003) Multimedia environments and interactive scientific presentation of industrially-based data sets. International Workshop on Design, Specification, and Verification of Interactive Systems, Berlin. 